\title{
Analysis of Net Causal Flows in Circuit of Premotor Control during Left Hand's Movement Readiness State
}

\author{
Yuqing Wang \\ Key Laboratory for NeuroInformation of Ministry of Education, \\ University of Electronic Science and Technology of China \\ Chengdu, P. R. China \\ Email: wyq112925@yahoo.cn \\ Ling Zeng and Huafu Chen \\ Key Laboratory for NeuroInformation of Ministry of Education, \\ University of Electronic Science and Technology of China \\ Chengdu, P. R. China \\ Email: lingz@uestc.edu.cn, chenhf@uestc.edu.cn
}

\begin{abstract}
-the previous research revealed some functional coupling among nodes in model of motor control in human brain, which described nondirectional synchronous actions among these nodes during movement-readiness state. However, causal relationships among these nodes, which represent some directional interactions in movementreadiness state, are still lack. In the present study, we used functional magnetic resonance imaging (fMRI) and conditional Granger causality (CGC) method to investigate the interactions in model of motor control in left hand's movement readiness state. Our results showed that upper precuneus (UPCU) and cingulated motor area (CMA) revealed net causal influences with contra lateral supplementary motor areas and contra lateral caudate nucleus during the left hand's movement-readiness state. The net causal flows among these nodes can construct a closed circuit, which is similar as the circuit found in monkey's brain and in human's brain in right hand's movement readiness state. This confirmed that there was an intrinsic circuit for motor control in either right hand's or left hand's movement readiness. Moreover, the results of Out-In degrees indicated that bilateral primary sensorimotor areas revealed competitive relationship during left hand's movement-readiness.
\end{abstract}

Index Terms-movement-readiness, conditional granger causality analysis

\section{INTRODUCTION}

The movement-readiness state defined as a premovement phase, which involved evolvement of cerebral networks from the resting state to movementexecution state, has been studied intensively for years using brain imaging techniques [1-3]. The previous study on monkey in right hand's movement readiness revealed a circuit of motor control passing through anterior cingulated cortex (ACC), posterior cingulated cortex (PCC), upper precuneus (UPCU), caudate nucleus (CN), cingulated motor area (CMA), left supplementary motor area (LSMA) and left primary sensorimotor area (LS1M1) in turn [4]. The movement-readiness state has been confirmed to involve in motor preparation, anticipation, restraint, execution and learning $[4,5]$. So, we assumed that widely interactions in model of motor control were employed for future movement in human brain, and we also assumed that there was a similar circuit in human brain during left hand's movement readiness state.

Using functional correlations analysis method, Newton et al. revealed significantly increased correlations within the sensorimotor networks (SMN) [1]. Fox and Raichles et al. found that default model network (DMN) and SMN were completely separated in resting state but anti-correlated in movement execution $[6,7]$. Furthermore, Treserras et al. found that PCC were aroused prominent functional coupling with UPCU, right supplementary motor area (RSMA) and right primary sensorimotor cortex (RS1M1); however ACC delivered the prominent functional coupling to LS1M1 in right hand's movementreadiness state [3]. These results just revealed the functional coupling in model of motor control in human brain; however, the directional causal flow in model of motor control is still lack.

Recently, there was a growing concern for interactions in model of motor control from the causal point of view. Using structural equation modeling (SEM), Solodkin et al. found that the input from supplementary motor area (SMA) to primary sensorimotor area (S1M1) was facilitated during movement execution (ME) [8]. Using dynamic causal modeling (DCM), Grefkes et al revealed enhanced neural coupling towards the contra lateral S1M1 but reduced towords ipsilateral S1M1 during unimanual ME [2]. There were some limitations in SEM and DCM methods, such as the requirement of prior directional connectivity information and low effects for multi-nodes situation. However, Granger causality (GC) method can effectively overcome these shortcomings. [9, 10]. Using GC method, Uddin et al found that PCC exerted greater 
influences on its anticorrelated networks [9]. Using GC method, Chen et al. found the forward and backward causal influences among SMA, the bilateral dorsal premotor area (PMd), the contra lateral primary and secondary somatosensory cortex (S1) and the primary motor cortex (M1) during motor imagery (MI) [10]. The GC method was just suitable for bi-variation situation. So, conditional Granger causality (CGC) method, which was an extend GC method, was proposed for multi-variation situation [7, 11-13]. Using CGC method, Liao et al found that self-referential network (SRN) exerted the strongest causal influences to other networks; however DMN was affected by other networks [14, 15]. These studies explored causal influences in model of motor control in ME or MI; however, the estimation of causal influences was still lack during movement-readiness state, and the existence of the whole dynamic circuit wasn't validated in human brain during left hand's movement readiness.

In the present study, CGC method was employed to resolve the aforementioned problems. Firstly, ACC, PCC, UPCU, left caudate nucleus (LCN), right caudate nucleus (RCN), CMA, LSMA, LS1M1, RSMA and RS1M1 were acquired by independent components analysis (ICA). CGC method was then employed to analyze the directed influence among these regions. Subsequently, the net CGC flows were acquired by subtracting bi-directional CGC values. Finally, the nonparametric bootstrap methodology was applied to assess the statistically significant threshold.

\section{MATERIAL AND METHODS}

\section{A. Subjects}

Sixteen healthy subjects (8 female and 8 male, mean ages 24.8) without history of neurological and psychiatric disorder participated in the fMRI experiment. All participants were confirmed as right-handed using the Edinburgh Handedness Inventory and had normal or corrected-to-normal vision. Written informed consent was given prior to scanning. The experiments were approved by the local ethics committee.

\section{B. Scanning procedures and tasks}

The experiment was performed on SIEMENS Trio 3T scanner, in Huaxi MR Research Center, Chengdu, China. The gradient-recalled echo planar imaging (EPI) sequence was employed for fMRI scanning and the parameters were as follows: 30 transverse slices, $\mathrm{TR}=$ 2000ms, $\mathrm{TE}=30 \mathrm{~ms}, \mathrm{FOV}=24 \mathrm{~cm}$, matrix $=64 \times 64$, voxel size $=3.75 \times 3.75 \times 5 \mathrm{~mm}$ (without gap), flip angle $=90^{\circ}$. Subjects were required to remain still and relax with keeping their eyes closed until heard an oral signal ("ready"); then waiting for an oral signal ("move") before executing task (left hand grasping with ratio of $2 \mathrm{~Hz}$ ); and finally, subjects were required to execute the sensorimotor task until heard the oral signal ("stop"). The whole tasks would also last 6 min 40 sec.

\section{Data preprocessing and choosing region of interest(ROI)}

The realignment was employed to reduce the effect of head motion. All realigned images were spatially normalized to the EPI template using registration. Spatial smoothing was employed to enhance the signal-to-noise ratio using a $6 \mathrm{~mm}$ cubic Gaussian kernel with a full width half-maximum (FWHM). All these operations were completed in SPM8 (www.fil.ion.ucl.ac.uk_spm). The group ICA of the smoothed datasets of movementreadiness of all subjects was carried out in GIFT (Group ICA of fMRI Toolbox) (http://icatb.sourceforge.net/) [16]. Based on previous published results, 10 components were chosen, which were corresponded respectively to LSMA, RSMA, CMA, LS1M1, RS1M1 PCC, UPCU, ACC, LCN and RCN $[3,5]$.

\section{CGC method}

Based on the mathematic description of the CGC, a measure of linear dependence was proposed, which could effectively calculate GC in light of vector autoregressive models [7, 17, 18]. Consider three time series Xt ,Yt and $\mathrm{Zt}$. Firstly, the joint autoregressive representation for $\mathrm{Xt}$ and $\mathrm{Zt}$ can be written as follow:

$$
\begin{aligned}
& X_{t}=\sum_{j=1}^{p} a_{1 j} X_{t-j}+\sum_{j=1}^{p} b_{1 j} Z_{t-j}+\varepsilon_{1 t} \\
& Z_{t}=\sum_{j=1}^{p} c_{1 j} X_{t-j}+\sum_{j=1}^{p} d_{1 j} Z_{t-j}+\varepsilon_{2 t}
\end{aligned}
$$

where the covariance matrix of the residual noise terms is:

$$
\sum 1=\left(\begin{array}{cc}
\operatorname{var}\left(\varepsilon_{1 t}\right) & \operatorname{cov}\left(\varepsilon_{1 t}, \varepsilon_{2 t}\right) \\
\operatorname{cov}\left(\varepsilon_{2 t}, \varepsilon_{1 t}\right) & \operatorname{var}\left(\varepsilon_{2 t}\right)
\end{array}\right)
$$

Then we consider the joint autoregressive representation of all three time courses $\mathrm{Xt}$, Yt and $\mathrm{Zt}$ :

$$
\begin{aligned}
& X_{t}=\sum_{j=1}^{p} a_{2 j} X_{t-j}+\sum_{j=1}^{p} b_{2 j} Y_{t-j}+\sum_{j=1}^{p} c_{2 j} Z_{t-j}+\varepsilon_{3 t} \\
& Y_{t}=\sum_{j=1}^{p} d_{2 j} X_{t-j}+\sum_{j=1}^{p} e_{2 j} Y_{t-j}+\sum_{j=1}^{p} f_{2 j} Z_{t-j}+\varepsilon_{4 t} \\
& Z_{t}=\sum_{j=1}^{p} g_{2 j} X_{t-j}+\sum_{j=1}^{p} h_{2 j} Y_{t-j}+\sum_{j=1}^{p} k_{2 j} Z_{t-j}+\varepsilon_{5 t}
\end{aligned}
$$

where the covariance matrix of the residual noise terms is:

$$
\sum 2=\left(\begin{array}{ccc}
\operatorname{var}\left(\varepsilon_{3 t}\right) & \operatorname{cov}\left(\varepsilon_{3 t}, \varepsilon_{4 t}\right) & \operatorname{cov}\left(\varepsilon_{3 t}, \varepsilon_{5 t}\right) \\
\operatorname{cov}\left(\varepsilon_{4 t}, \varepsilon_{3 t}\right) & \operatorname{var}\left(\varepsilon_{4 t}\right) & \operatorname{cov}\left(\varepsilon_{4 t}, \varepsilon_{5 t}\right) \\
\operatorname{cov}\left(\varepsilon_{5 t}, \varepsilon_{3 t}\right) & \operatorname{cov}\left(\varepsilon_{5 t}, \varepsilon_{4 t}\right) & \operatorname{var}\left(\varepsilon_{5 t}\right)
\end{array}\right)
$$

where $\mathrm{p}$ is the order of the autoregressive model; and $\varepsilon_{i t}, i=1,2,3,4,5$ are the uncorrelated prediction error over time. From these two sets of equations, we define the conditional Granger causality from time-course Yt to Xt conditional on time-course $\mathrm{Zt}$ as:

$$
F_{Y \rightarrow X \mid Z}=\ln \left(\frac{\operatorname{var}\left(\varepsilon_{1 t}\right)}{\operatorname{var}\left(\varepsilon_{3 t}\right)}\right)
$$

When the causal influence from time-course Yt to $\mathrm{Xt}$ is entirely due to other time course $\mathrm{Zt}$, the coefficients $\mathrm{b} 2 \mathrm{j}$ in Eq.3 are uniformly zero, and $\operatorname{var}\left(\varepsilon_{1 t}\right)=\operatorname{var}\left(\varepsilon_{3 t}\right)$. So, $F_{Y \rightarrow X \mid Z}=0$ meaning that no further improvement in the 
predication of time-course $\mathrm{Xt}$ can be expected by including past measurements of time-course $\mathrm{Yt}$ conditioned on the other time-course $\mathrm{Zt}$. In contrast, when a direct influence from time-course Yt to Xt exists, the inclusion of past measurements of time-course Yt in addition to that of time-course $\mathrm{Xt}$ and $\mathrm{Zt}$ should result in better predictions of time-course $\mathrm{Xt}$, leading to $\operatorname{var}\left(\varepsilon_{1 t}\right)>\operatorname{var}\left(\varepsilon_{3 t}\right)$, and $F_{Y \rightarrow X \mid Z}>0$ [12]

The order of the autoregressive model was set to 6 using the Schwarz criterion (SC). The coefficients of the models were calculated using a standard least squares optimization.

\section{E. Calculating net Causal flows among ROIs}

The time course of each ROI was generated by averaging time courses of all voxels in ROI. Linear drift and linear regression were employed to eliminate the gross signal drifts, gross physiological changes, the artifacts of head moving, the interference from the signal of cerebrospinal fluid (CSF), white matter and individual response component, aiming to the state of movementexecution. CGC were employed to calculate the net causal flows among chosen ROIs during movement readiness state, using our programs coded in MATLAB (The Mathworks, Natick, MA) in terms of previous studies $[17,18]$. The net direct flows as follow:

$$
F_{N E T \mid Z}=F_{Y \rightarrow X \mid Z}-F_{X \rightarrow Y \mid Z}
$$

To assess the statistical significance of CGC results, a nonparametric estimation based on the bootstrap methodology was applied to obtain the null distribution [19].

\section{RESULTS}

\section{A. Choosing ten nodes}

The Fig.1 revealed the spatial distribution for ten ROIs with a statistic threshold. The Table. 1 enumerated the Brodmann area and the Talairach coordinates of peak voxels of ten nodes chosen by group ICA.

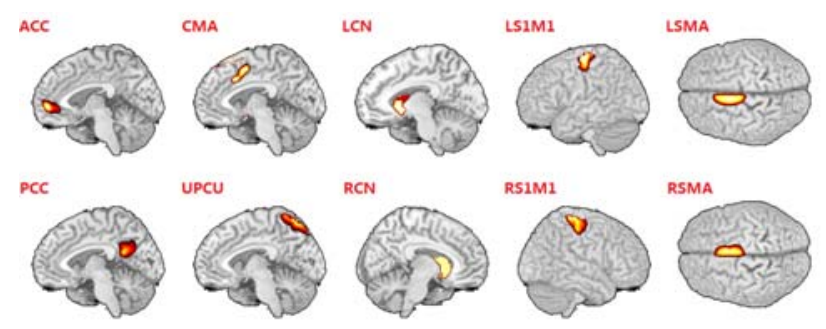

Figure 1. The location map of ten ROIs.
TABLE I.

THE DETAIL INFORMATION OF TEN ROIS

\begin{tabular}{|c|c|c|}
\hline Node's name & Brodmann area & Coordinate \\
\hline ACC & $10,24,32$ & $-2,55,1$ \\
\hline CMA & $6,24,32$ & $1,15,39$ \\
\hline LCN & $/$ & $-12,15,6$ \\
\hline LS1M1 & $2,3,4$ & $-38,-19,65$ \\
\hline LSMA & 6 & $-10,2,60$ \\
\hline PCC & 23,30 & $-1,-50,20$ \\
\hline UPCU & 7 & $-1,-67,58$ \\
\hline RCN & $/$ & $12,13,10$ \\
\hline RS1M1 & $2,3,4$ & $35,-34,68$ \\
\hline RSMA & 6 & $4,10,49$ \\
\hline
\end{tabular}

\section{B. Net causal flows in left hand's movement-readiness} state

The Fig.2 revealed the map for CGC in left hand's movement-readiness state. The Table.2 enumerated the detail of the mean of CGC across sixteen subjects. Each row meant $F_{x \rightarrow y \mid z}$ and each column meant $F_{y \rightarrow x \mid z}$. Some nodes (especially CMA and UPCU) revealed lateralization of interaction in left hand's movementreadiness state. CMA revealed net causal influences with RCN and RSMA, UPCU exerted net causal directional influence to RCN at the same time.

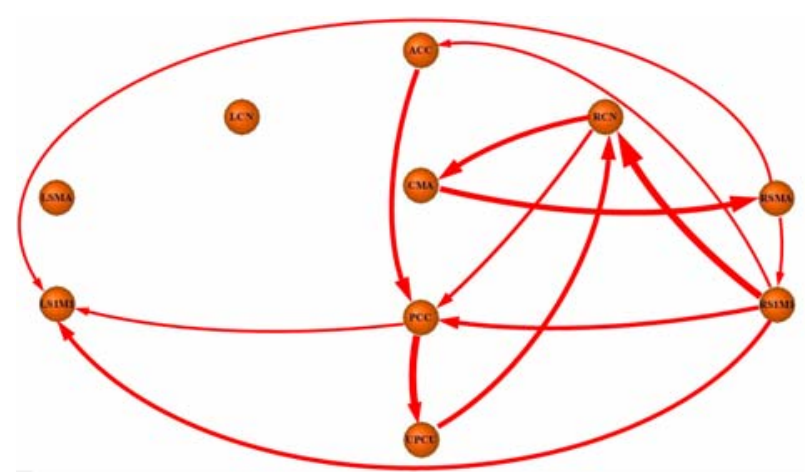

Figure 2. The map of net causal influences in left hand's movementreadiness state

TABLE II.

THE VALUES OF CGC DURING LEFT HAND’S MOVEMENT-READINESS

\begin{tabular}{|c|c|c|c|c|c|c|c|c|c|c|}
\hline & 1 & 2 & 3 & 4 & 5 & 6 & 7 & 8 & 9 & 10 \\
\hline 1 & $/$ & $/$ & $/$ & $/$ & $/$ & .007 & $/$ & $/$ & $/$ & $/$ \\
\hline 2 & $/$ & $/$ & $/$ & $/$ & $/$ & $/$ & $/$ & $/$ & $/$ & .008 \\
\hline 3 & $/$ & $/$ & $/$ & $/$ & $/$ & .005 & $/$ & $/$ & $/$ & $/$ \\
\hline 4 & $/$ & $/$ & $/$ & $/$ & $/$ & $/$ & $/$ & $/$ & $/$ & $/$ \\
\hline 5 & $/$ & $/$ & $/$ & $/$ & $/$ & $/$ & $/$ & $/$ & $/$ & $/$ \\
\hline 6 & $/$ & $/$ & $/$ & .004 & $/$ & $/$ & .01 & $/$ & $/$ & $/$ \\
\hline 7 & $/$ & $/$ & $/$ & $/$ & $/$ & $/$ & $/$ & .007 & $/$ & $/$ \\
\hline 8 & $/$ & .007 & $/$ & $/$ & $/$ & $/$ & $/$ & $/$ & $/$ & $/$ \\
\hline 9 & .004 & $/$ & $/$ & .006 & $/$ & .006 & $/$ & .01 & $/$ & $/$ \\
\hline 10 & $/$ & $/$ & $/$ & .004 & $/$ & $/$ & $/$ & $/$ & .004 & $/$ \\
\hline
\end{tabular}

1=ACC, 2= CMA, 3=LCN, 4=LS1M1, 5=LSMA, 6=PCC, 7=UPCU, 8=RCN, 9=RS1M1, 10=RSMA 


\section{Out-In degrees of ten nodes in left hand's movement readiness state}

The Fig. 3 revealed the map of Out-In degrees of ten nodes in left hand's movement-readiness state. In the Fig.3, RS1M1 revealed highest positive Out-In degrees, however, LS1M1 revealed highest negative Out-In degree

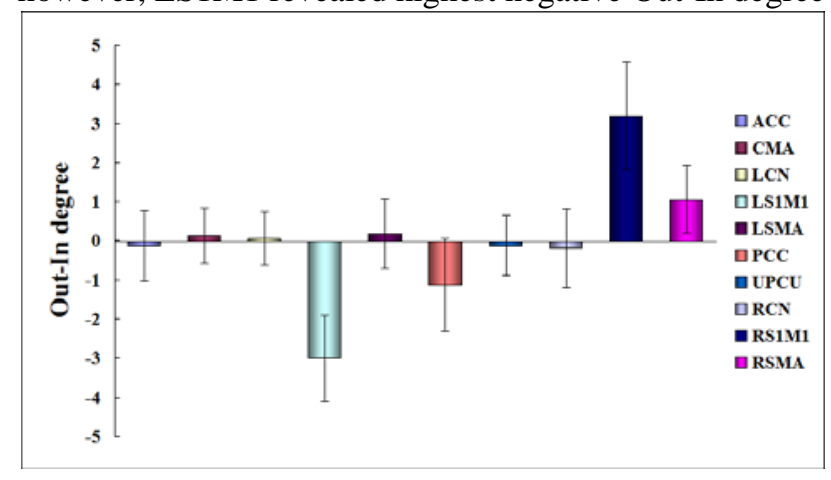

Figure 3. The map of net causal influences in left hand's movementreadiness state

\section{A circuit in left hand's movement readiness state}

The Fig.4 revealed a circuit during left hand's movement readiness state, which was consisted with the circuit in monkey's brain and was similar but mirrorimage as that found during right hand's movement readiness state. The circuit passed through seven nodes as follow:

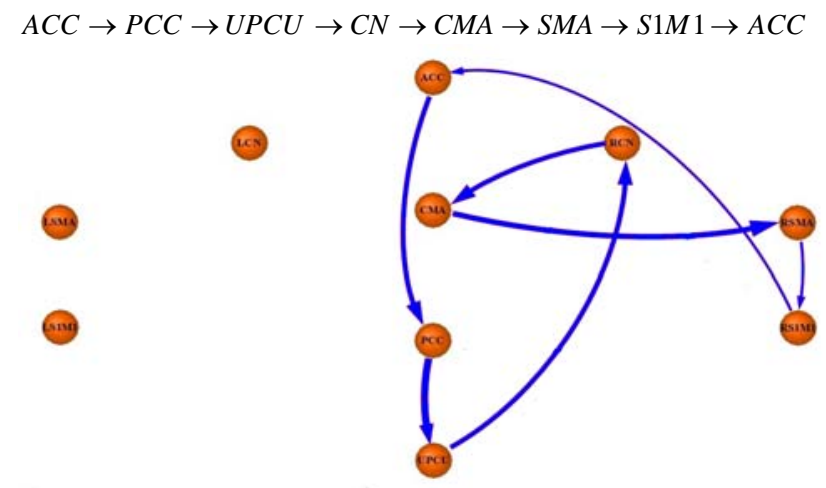

Figure 4. The map of a circuit during left hand's movement readiness state

\section{DISCUSSION}

In the present study, the CGC method was applied successfully to investigate the interactions among ten nodes involving motor control in left hand's movementreadiness state. Our result showed that CMA revealed net causal influences with nodes in right SMN and in right corpus striatum, UPCU revealed net causal influence with node in right corpus striatum. Based on net causal flows among nodes, we found a circuit which was similar but enantiomorphous to that found in right hand's movement readiness state. Moreover, the results of Out-In degrees indicated that RS1M1 preferred modulating other nodes, in contrast, LS1M1 preferred being affected by other nodes in circuit of motor control during left hand's movement-readiness state.

\section{A. Net causal influences during left hand's movement readiness state}

The net uni-directional influence from UPCU to RCN was consisted with the previous research in monkey corticocortical connections [3, 5, 20, 21]. The limbic system upstream was anatomically connected by UPCU with the sensorimotor system downstream which involved in hand motor representation [5, 22]. Moreover, UPCU was also related to mental motor imagery, spatial control of hand movement, generating successively underway movement in terms of buffered memory [2326]. Treserras et al assumed that the UPCU devoted to multimodal sensory integration [3]. $\mathrm{CN}$ was an authorization structure for motor control which received the information of premotor processed sent by limbic system, and then processed the information at highest cognitive level [4, 27-29]. So, in the present study, net causal influence from UPCU to RCN indicated that RCN collected initiatory messages sending by UPCU during left hand's movement-readiness [4, 30]. The net unidirectional influence from RCN to CMA and that from CMA to RSMA were consisted with the previous research in monkey corticocortical connections [3, 5, 20, 21]. In fact, CMA was a key region associating with response and choice of optimal planning and task control function [4, 27, 30, 31-33]. The potential mechanism was that CMA received numerous optimal plans for movement from RCN; then generating a reasonable response selection for left hand's movement; and finally sent a facilitated signal to RSMA during left hand's movement-readiness [4, 20, 21].

\section{B. A circuit in left hand's movement readiness state}

Firstly, our results revealed that net causal flows could construct a circuit as follow:

$A C C \rightarrow P C C \rightarrow U P C U \rightarrow R C N \rightarrow C M A \rightarrow R S M A \rightarrow R S 1 M 1 \rightarrow A C C$. The circuit was similar to that found in monkey corticocortical connections and was also similar but mirrorimage as that found in right hand's movement readiness [3, 5]. So, the circuit confirmed our hypothesis in previous research from view of dynamic causality. The ACC as part of the rostral limbic system was a key region generating behavior predictions and guiding our actions, playing emotive and motivational role in motor behavior [20, 21, 34-36]. Hence, ACC transmitting net causal flow to PCC suggested that ACC generated initialized information flowing to the PCC [37-40]. PCC was a centre which play important role in reviewing past knowledge [5, 41]. The potential mechanism for the net causal flow from PCC to UPCU might be that PCC reviewed fix behavior pattern and sent an initialization to UPCU for movement readiness. The UPCU was an intermedia region which connects anatomically with the limbic system upstream and with the sensorimotor system downstream treating of hand motor representation [5, 22]. Moreover, UPCU involved in mental MI, spatial control of hand movement, generating successively underway movement in terms of buffered memory [23-26]. Treserras et al. thereby suggested that UPCU devoted to multimodal sensory integration and found UPCU aroused 
functional connectivity with PCC [3]. In the present study, the net causal flow from UPCU to RCN suggested that RCN collected initiatory messages sent by UPCU $[5,30]$. The caudate nucleus (CN) was an authorization structure for motor control which received the information of premotor processed by limbic system and then processed the information at highest cognitive level [5, 27-29]. The net causal flow from RCN to CMA suggested information communication for response and choice of optimal planning [5, 27, 30]. CMA was a key region associated with task control function [31-33]. The potential mechanism of net causal flow from CMA to RSMA was that CMA received numerous optimal plans for movement from RCN, then generating a reasonable response selection for left hand's movement, and finally sent a facilitated signal to RSMA [5, 21]. Due to the SMA was related to motor output, the net causal flow from RSMA to RS1M1 was regarded as the facilitation during left hand's movement readiness [2, 42, 43]. Finally, the net causal flow from RS1M1 to ACC implied that the initial information sent by ACC was returned to ACC by passing through upmentioned closed circuit in the present study. This feedback might suggest a reinforced process for the currency of information flow in the circuit. In general, this circuit was consisted with most parts of circuit in monkey study and was similar but mirrorimage as that found in human brain during right hand's movement readiness. This result suggested that there was an intrinsic circuit for motor control during either right hand's or left hand's movement readiness. The intrinsic circuit can be described as follow:

$A C C \rightarrow P C C \rightarrow U P C U \rightarrow C N \rightarrow C M A \rightarrow S M A \rightarrow S 1 M 1 \rightarrow A C C$.

In addition, there were other causal flows which could be regarded as an inhibitory role for task demand in the present study. The net causal flow from RSMA to LS1M1, which was consisted with the previous results, might imply an inhibitory process from RSMA to LS1M1 during left hand's movement readiness [2]. At the same time, the previous functional connectivity research indicated that coupling between RS1M1 and LS1M1 decreased, whereas coupling between RS1M1 and RSMA increased during left hand's movement-readiness state [1, 3]. Our net causal flow revealed that RS1M1 received net causal flow from RSMA and transmitted net causal flow to LS1M1, suggesting potential modulations of coupling between RS1M1 and RSMA during left hand's movement readiness [10, 44-47]. The previous results suggested that PCC aroused enhanced functional connectivity with RS1M1, implying some interactions among two nodes in right hand's movement readiness state [3, 48]. Our results showed that PCC received significant net causal flow from RS1M1 and transmitted significant net causal flow to LS1M1 in left hand's movement readiness state, providing an indirectly evidence for the upmentioned suggestion. In addition, these results also indicated that PCC as a relay station transmitted the inhibitory information from one side of SMN to another side during unimanual behaviors. This suggestion might imply the potential mechanism that PCC mediated the coupling between SMN in two hemispheres for task demand. In general, based on net causal flows among nodes, we found a circuit which was similar but mirrorimage as that found in right hand's movement readiness state. The result suggested that there was an intrinsic circuit for motor control during either right hand's or left hand's movement readiness. In additional, our findings revealed more interactions among nodes in human brain than in monkey brain. The potential mechanism might attribute to more complex cognitive processing and more modulation among nodes in the circuit for robusticity of the circuit $[3,9,26,35,36,49$, 50].

\section{Out-In degrees of ten nodes in left hand's movement- readiness state}

In the Fig.3, highest negative Out-In degrees indicated that LS1M1 preferred being affected by other nodes in circuit of motor control during left hand's movementreadiness. In the present study, LS1M1 was exerted net uni-directional influence by RS1M1, RSMA and PCC. The net influences from RSMA and from RS1M1 to LS1M1 were consisted with the previous results, implying inhibitory modulations from right SMN to LS1M1 during left hand's movement-readiness [2]. The net influence from PCC to LS1M1, which was consisted with the inference of the previous study on functional connectivity between PCC and RS1M1, suggested that PCC mediated the coupling between SMN in two hemispheres for task demand in the present study [3]. In the Fig.3, highest positive Out-In degrees indicated that RS1M1 preferred modulating other nodes in circuit of motor control during left hand's movement-readiness. In the present study, RS1M1 exerted net uni-directional influences to LS1M1, ACC, RCN and PCC. The net influence from RS1M1 to PCC suggested that RS1M1 suppressed PCC for task demand in the present study [3]. The previous research revealed that RCN functionally coupled with RS1M1 during the resting state [51]. In the present study, the net influence from RS1M1 to RCN could be regarded as a part of a closed local circuit as follow $R C N \rightarrow C M A \rightarrow R S M A \rightarrow R S 1 M 1 \rightarrow R C N$. This result might suggest that RS1M1 exerted facilitated modulation to RCN for the currency of the circuit during left hand's movement-readiness.

\section{CONCLUSION}

In the present study, fMRI and CGC were adopted successfully to investigate the interactions among ten nodes involving motor control in left hand's movement readiness state. Our result revealed that a net causal flow passed through UPCU, RCN, CMA and RSMA in turn during left hand's movement-readiness. Based on net causal flows among nodes, we found a circuit which was similar but enantiomorphous to that found in right hand's movement readiness state. The result suggested that there was an intrinsic circuit for motor control during either right hand's or left hand's movement readiness. Moreover, the results of Out-In degrees indicated that bilateral primary sensorimotor areas revealed competitive relationship during unimanual movement-readiness. 


\section{ACKNOWLEDGMENT}

This work is supported by the Natural Science Foundation of China (61035006, 30900326 and 90820006). And supported by Program for Chang jiang Scholars and Innovative Research Team in University

\section{REFERENCES}

[1] A.T. Newton, V.L. Morgan, J.C. Gore, "Task demand modulation of steady-state functional connectivity to primary motor cortex”, Hum Brain Mapp, vol. 28, pp. 663672, 2007.

[2] C. Grefkes, S. B. Eickhoff, D. A. Nowak, D. Manuel, R.F. Gereon, "Dynamic intra- and interhemispheric interactions during unilateral and bilateral hand movements assessed with fMRI and DCM”, Neuroimage, vol. 41, pp.1382-94, 2008.

[3] S. Treserras, K. Boulanouar, F. Conchou, M.S. Moreau, I. Berry, P. Celsis, F. Chollet, I. Loubinoux, "Transition from rest to movement: brain correlates revealed by functional connectivity”, Neuroimage, vol. 48, pp.207-216, 2009.

[4] M.P. Deiber, V. Ibanez, N. Sadato, M. Hallett. "Cerebral structures participating in motor preparation in humans: a positron emission tomography study. J Neurophysiol 75:233-247. 1996

[5] V. B. Brooks, "The neural basis of motor control", Oxford University Press, Oxford, 1986.

[6] M. D. Fox, A. Z. Snyder, J. L. Vincent, M. Corbetta, D.C. Van Essen, M.E. Raichle, "The human brain is intrinsically organized into dynamic, anticorrelated functional networks”, Proc Natl Acad Sci USA, vol.102, pp.96739678, 2005.

[7] M.E. Raichle, A.Z. Snyder, 2007. "A Default Mode of Brain Function: A Brief History of an Evolving Idea”, NeuroImage 37:1083-1090.

[8] A. Solodkin, P. Hlustik, E. E. Chen, S.L. Small, "Fine modulation in network activation during motorexecution and motor imagery”, Cereb Cortex, vol. 14, pp.1246-1255, 2004.

[9] L. Q. Uddin, A. M. C. Kelly, B. B. Biswal, F.X. Castellanos, M.P. Milham, "Functional connectivity of default mode network components: correlation, anticorrelation, and causality”, Hum. Brain Mapp. vol. 30, pp.625-637, 2009.

[10] H. Chen, Q. Yang, W. Liao, Q. Gong, S. Shen, "Evaluation of the effective connectivity of supplementary motor areas during motor imagery using Granger causality mapping”, Neuroimage 47: 1844-1853, 2009.

[11] J.F. Geweke,. "Measures of conditional linear dependence and feedback between time series”, J Am Stat Assoc 79:709-715, 1984.

[12] Y. Chen, S.L. Bressler, M. Ding, "Frequency decomposition of conditional Granger causality and application to multivariate neural field potential data”, J Neurosci Methods 150:228-237, 2006.

[13] Z. Zhou, Y. Chen, M. Ding, P. Wright, Z. Lu, Y. Liu, "Analyzing brain networks with PCA and conditional Granger causality”, Hum. Brain Mapp. 30, 2197-2206, 2009.
[14] M. Ding, Y. Chen, and S. L. Bressler, "Granger Causality: Basic Theory and Application to Neuroscience", Verlage: Wiley-VCH, 2006.

[15] W. Liao, D. Mantini, Z. Zhang, Z. Pan, J. Ding, Q. Gong, Y. Yang, $\mathrm{H}$. Chen, "Evaluating the effective connectivity of resting state networks using conditional Granger causality”, Biol Cybern. vol. 102, pp.57-69, 2009.

[16] V. D. Calhoun, T. Adali, G. D. Pearlson, J.J. Pekar, “A method for making group Inferences from functional MRI data using independent component analysis”, Hum Brain Mapp, vol. 14, pp.140-151, 2001.

[17] R. Goebel, A. Roebroeck, D. Kim, E. Formisano, "Investigating directed cortical interactions in timeresolved fMRI data using vector autoregressive modeling and Granger causality mapping”, Magn. Reson. Imaging, vol. 21, pp.1251-1261, 2003.

[18] A. Roebroeck, E. Formisano, and R. Goebel, "Mapping directed influence over the brain using Granger causality and fMRI”, NeuroImage, vol. 25, pp.230-242, 2005.

[19] B. Efron, R. J. Tibshirani, "An Introduction to the Bootstrap”, Chapman and Hall, NewYork, 1993.

[20] W. Schultz, P. Apicella, E. Scarnati, T. T Ljungberg, "Neuronal activity in monkey ventral striatum related to the expectation of reward”, J Neurosci, vol. 12, pp.45954610, 1992.

[21] O. Devinsky, M. J. Morrell, and B. A. Vogt, "Contributions of anterior cingulate cortex to behaviour", Brain, vol. 118, pp.279-306, 1995.

[22] J. Parvizi, G. W. Van Hoesen, J. Buckwalter, A. Damasio, "Neural connections of the posteromedial cortex in the macaque”, Proc Natl Acad Sci USA, vol. 103, pp.15631568, 2006.

[23] N. Sadato, G. Campbell, V. Ibanez, M.P. Deiber, M. Hallett, "Complexity affects regional cerebral blood flow change during sequential finger movements", J Neurosci, vol. 16, pp.2691-2700, 1996.

[24] T. Ogiso, K. Kobayashi. and M. Sugishita, "The precuneus in motor imagery: a magnetoencephalographic study", Neuroreport, vol.11, pp.1345-1349, 2000.

[25] K. Oishi, K. Toma, E. Bagarinao, K. Matsuo, T. Nakai, K. Chihara, H. Fukuyama, "Activation of the precuneus is related to reduced reaction time in serial reaction time tasks”, Neurosci Res, vol.52, pp.37-45, 2005.

[26] A. E. Cavanna, and M. R. Trimble, “The precuneus: a review of its functional anatomy and behavioural correlates”, Brain, vol.129, pp.564-583, 2006.

[27] S. N. Haber, "The primate basal ganglia: parallel and integrative networks”, J Chem Neuroanat., vol. 26, pp.317330, 2003

[28] S. Lehericy, E. Bardinet, L.Tremblay, P.F. Van de Moortele, J.B. Pochon, D. Dormont, D.S. Kim, J. Yelnik, K. Ugurbil, "Motor control in basal ganglia circuits using fMRI and brain atlas approaches", Cereb Cortex, vol.16, pp. 149-161, 2006.

[29] A. Martino, A. Scheres, D. S. Margulies, A.M.C. Kelly, L.Q. Uddin, Z. Shehzad, B. Biswal, J.R. Walters, F.X. Castellanos, M.P. Milham, "Functional Connectivity of Human Striatum: A Resting State fMRI Study", Cereb Cortex, vol.18, pp.2735-2747, 2008.

[30] E. T. Rolls, "Neurophysiology and cognitive functions of the striatum”, Rev Neurol (Paris), vol.150, pp.648-660, 1994. 
[31] N. U. Dosenbach, K. M. Visscher, E. D. Palmer, F.M. Miezin, K.K. Wenger, H.C. Kang, E.D. Burgund, A.L.

[32] Grimes, B.L. Schlaggar, S.E. Petersen, “A core systemfor the implementation of task sets”, Neu-ron, vol.50, pp.799812, 2006.

[33] N.U. Dosenbach, D. A. Fair, F. M. Miezin, A.L. Cohen, K.K. Wenger, R.A.T. Dosenbach, M.D. Fox, A.Z. Snyder, J.L. Vincent, M.E. Raichle, B.L. Schlaggar, S.E. Petersen, "Distinct brain networks for adaptive and stable task control in humans", ProcNatlAcad Sci USA, vol.104, pp.1073-11078, 2007.

[34] D. Mantini, M. Corbetta, M.G. Perrucci, M.G. Perruccia, G.L. Romania, C.D. Gratta, "Large-scale brain networks account for sustained and transient activity during target detection”, Neuroimage, vol.44, pp.265-274, 2009.

[35] K.F. Muakkassa, P.L Strick,. "Frontal lobe inputs to primate motor cortex: evidence for four somatotopically organized 'premotor' area”, Brain Res. 177, 176-182, 1979.

[36] C. Summerfield, T. Egner, M. Greene, E, Koechlin, J. Mangels, J. Hirsch, "Predictive codes for forthcoming perception in the frontal cortex", Science 314: 1311-1314, 2006.

[37] M. Bar, E. Aminoff, M. Mason, M. Fenske, "The units of thought”, Hippocampus 17: 420-428, 2007.

[38] C. Baleydier, F. Mauguiere, "The duality of the cingulate gyrus in monkey. Neuroanatomical study and functional hypothesis”, Brain 103: 525-554, 1980.

[39] B.A. Vogt, D.N. Pandya, D.L. Rosene, "Cingulate cortex of the rhesus monkey: I. Cytoarchitecture and thalamic afferents”, J Comp Neurol 262: 256-270, 1987.

[40] H. Tokuno, A. Nambu, “Organization of nonprimary motor cortical inputs on pyramidal and nonpyramidal tract neurons of primary motor cortex: An electrophysiological study in the macaque monkey”, Cereb Cortex 10: 58-68, 2000.

[41] J.L. Vincent, G.H. Patel, M.D. Fox, A.Z. Snyder, J.T. Baker, D.C. Van Essen, J.M. Zempel, L.H. Snyder, M. Corbetta, M.E. Raichle, Intrinsic functional architecture in the anaesthetized monkey brain”, Nature 447: 83-86, 2007.

[42] S.V. Astafiev, G.L. Shulman, C.M. Stanley, A.Z. Snyder, D.C. Van Essen, M. Corbetta, "Functional organization of human intraparietal and frontal cortex for attending looking and pointing”, J Neurosci 23: 4689-4699, 2003.

[43] P. Nachev, H. Wydell, K. O'Neill, M. Husain, C. Kennard, "The role of the pre-supplementary motor area in the control of action”, Neuroimage 36 Suppl 2: T155-163, 2007.

[44] P. Nachev, C. Kennard, M. Husain, "Functional role of the supplementary and pre-supplementary motor areas", Nature Rev Neurosci. 9, 856-869, 2008.

[45] T. Baumer, F. Bock, G. Koch, R. Lange, J.C. Rothwell,, H.R. Siebner, A. Munchau, "Magnetic stimulation of human premotor or motor cortex produces interhemispheric facilitation through distinct pathways”, J Physiol 572: 857-868, 2006.

[46] C.H. Kasess, C. Windischberger, R. Cunnington, R. Lanzenberger, L. Pezawas, E. Moser, "The suppressive influence of SMA on M1 in motor imagery revealed by fMRI and dynamic causal modeling”, NeuroImage 40, 828-837, 2008.

[47] J. Reis, O.B. Swayne, Y. Vandermeeren, M. Camus, M.A. Dimyan, M. Harris-Love, M.A. Perez, P. Ragert, J.C.
Rothwell, L.G. Cohen, "Contribution of transcranial magnetic stimulation to the understanding of cortical mechanisms involved in motor control”, J Physiol 586: 325-351, 2008.

[48] Q. Gao, H. Chen, Q. Gong, "Evaluation of the effective connectivity of the dominant primary motor cortex during bimanual movement using Granger causality”, Neurosci Lett 443:1-6, 2008.

[49] M.F. Mason, M.I. Norton, J. D. Van Horn, D.M. Wegner, S.T. Grafton, C.N. Macrae, "Wandering minds: the default network and stimulus-independent thought", Science 315: 393-395, 2007.

[50] M.D. Greicius, V. Menon, "Default-mode activity during a passive sensory task: Uncoupled from deactivation but impacting activation”, J Cogn Neurosci 16:1484-1492, 2004.

[51] M.D. Greicius, B. Krasnow, A.L. Reiss, V. Menon, "Functional connectivity in the resting brain: a network analysis of the default mode hypothesis", Proc Natl Acad Sci U S A 100: 253-258, 2003.

[52] H. Boecker, J. Jankowski, P. Ditter et al, "A role of the basal ganglia and midbrain nuclei for initiation of motor sequences”, NeuroImage, vol. 39, pp.1356-1369, 2008.

Yuqing Wang is doctoral student of the

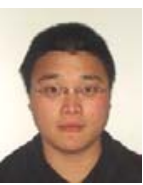
University of Electronic Science and Technology of China, He received the master degree in biophysics from the University of Electronic Science and Technology of China (UESTC), Chengdu, China, in 2006. He is the author or coauthor of the included MRI etc 6 papers. His current interests include fMRI and their applications in cognitive science and neurological problems.

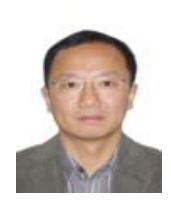

Ling Zeng $\mathbf{P h D}$ is professor and doctor teacher of he University of Electronic Science and Technology of China, He received the Ph.D. degree in biomedical engineering from the University of Electronic Science and Technology of China (UESTC), Chengdu, China, in 2008. He is the author or coauthor of the included MRI etc 20 papers. His current interests include fMRI and their applications in cognitive science and neurological problems.

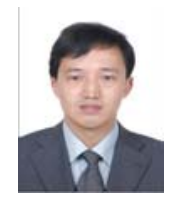

Huafu Chen PhD is professor and doctor teacher of the University of Electronic Science and Technology of China, He received the Ph.D. degree in biomedical engineering from the University of Electronic Science and Technology of China (UESTC), Chengdu, China, in 2004. , He had been a visiting scholar at University of Texas at San Antonio, USA. He is the author or coauthor of the included NeuroImage, IEEE Tran BME and IEEE Tans. MI etc 70 papers. His current interests include fMRI, EEG, and their applications in cognitive science and neurological problems. 